\title{
Depictions of motion devised by a blind person
}

\author{
JOHN M. KENNEDY and CYNTHIA E. MERKAS \\ University of Toronto, Scarborough, Ontario, Canada
}

\begin{abstract}
A blind man (E.A.) was asked to draw pictures suggesting wheels in various kinds of motion. Six pictures were drawn by E.A. The pictures were shown to sighted subjects, who were asked to assign labels to the pictures, in a multiple-choice format. The labels were assigned at a rate above chance. We argue that the pictures are metaphoric and that pictorial metaphor relies on common properties of the static picture and the kinetic referent.
\end{abstract}

In this article, we investigate whether nonrealistic devices for showing motion in a static picture devised by a blind man, at our request, can be understood by sighted people.

In some previous studies on tactile pictures, blind people have been asked to invent devices for showing motion (and many other problematic referents of static outline pictures, such as pain), but the recognizability of the drawings that resulted has not been tested. In other studies, blind people were tested with motion devices invented by the sighted. Since the pictures to be tested were devised by sighted investigators - albeit, investigators familiar with pictures drawn by blind people-it is not clear whether pictures devised by the blind would be widely interpretable. Even though the sighted investigators were influenced by some pictures drawn by the blind, there is no way of knowing to what extent the selection of test pictures represented what a blind person would produce. In short, a bias may have been introduced inadvertently by the sighted investigators. In the present study, the possibility of bias in the selection was diminished, since each picture drawn by the blind person was tested.

Our point about inadvertent effects will likely be clearer if we show how the kind of bias with which we were concerned could operate. From the multitude of tactile pictures known to sighted experimenters, previous investigators may have selected a microscopic fraction that they thought would make sense to both the blind and the sighted (say 5 drawings out of 3,000, with those 5 all being by entirely different blind people). That fraction of the population of drawings by the blind might have been produced essentially by chance by the blind people. As a result, a good deal of what was not chance in those studies might best be attributed to the selection process and the intervening role of the sighted investigators. For certain, those studies cannot tell us whether a blind person

We thank Esref Armagan and Joan Eroncel for essential aid in this research, Juan Bai for statistics advice, and Claudia Carello and the journal's reviewers for helpful commentary. Correspondence concerning this article should be addressed to J. M. Kennedy, University of Toronto, 1265 Military Trail, Scarborough, ON M1C 1A4, Canada (e-mail: kennedy@scar.utoronto.ca). could make up a set of pictorial devices, with restricted objectives, that would communicate successfully at a significant rate to the sighted.

At this point, a brief introduction to the literature on raised pictures for touch and the blind may be helpful to many readers. Raised pictures for tactile exploration by blind people have been devised for over 200 years. Educators and philosophers often argued that many of the representational devices in the pictures would have to be explained to the blind (Eriksson, 1998, ch. 4; Holmes, Hughes, \& Jansson, 1998). However, a mobile observer can explore an environment to discover the shapes of quite complex objects. Raised pictures can capitalize on the spatial knowledge garnered by touch. Furthermore, drawings made by the blind can depict aspects of the shapes of large objects shown reduced in scale, as well as those of small objects shown life-sized (Heller, 1989; Kennedy, 1997; Millar, 1991, 1994, 1997). Blind children 8-13 years of age can recognize raised-line drawings at a rate comparable with or better than that of sighted children attempting the task while blindfolded (D'Angiulli, Kennedy, \& Heller, 1998). Changes in the location of the observer's vantage point on a scene comprising three objects on a table top are reflected appropriately in drawings of the scene by the blind (Heller \& Kennedy, 1990).

Kennedy (1993) reported studies on the drawings of blind children and adults using a raised-line drawing kit. The kit contained a plastic sheet and a stiff board with a rubberized surface. When a ballpoint pen wrote on the sheet and the sheet was resting on the board, the result was a raised line. The line was raised on the side from which pressure was applied.

Some of the pictures made by blind subjects, Kennedy (1993) noted, included deliberate distortions of shapes. One blind boy ( 10 years of age, blind since age 2 ) was asked to draw a static wheel and a wheel rolling along. To depict the static wheel, he drew a circular wheel with spokes radiating from the hub. To show the wheel rolling along, he drew the wheel as an oval, more than twice as wide as it was tall, and he arranged the spokes in an irregular line, crossing over each other, along the major axis of the oval. Kennedy (1993) argued that this imaginative sketch was a metaphoric picture. 
Kim and Francis (1998) noted that lines trailing behind an object depicted as moving are often described as metaphoric. They devised a program for producing such lines behind representations of moving objects, and they suggested that the program's results might be a model for some effects, such as blur from motion in visual physiology.

Rosenblum, Saldana, and Carello (1993) argued that motion devices can give a sense of the path or style of movement, and since "events can be identified by the form of the flow" (p. 395), the devices can be related in a nonarbitrary way to dynamic events. They suggested that lines drawn as trailing from the backs of objects could be taken to be records of paths of movement. They called these streak lines. Displays with streak lines can be compared with long-duration exposures of objects to which some lights have been attached. Hence, they are related to optical structures and distinctive patterns that specify the shapes of distal sources and their dynamics (Arnheim, 1974; Friedman \& Stevenson, 1980; Runeson \& Frykholm, 1981).

Note that the wake lines behind a duck on water are ecologically valid and can help specify the motion of an object. They are a distinctive pattern arising in nature from the movement of an object across a nonrigid surface. The same lines depicted in the sky behind a flying bird may be understood by the observer to be borrowed from the situation in which they are ecologically valid and applied where they are not found in nature. On the one hand, their distinctiveness allows them to be informative. On the other, their unusual location violates the laws of nature and is understood to be metaphoric.

Streak lines are similar to some wake lines, tracks left in muddy conditions, the clearing left by a cloth sweeping across a dusty surface, trampled vegetation where a heavy animal has passed, ribbons flying from the back of a moving object, and other indicators of motion paths. But putting lines corresponding to the edges of these records in the otherwise empty air behind an object is metaphoric. Similarly, some changes in the shapes of parts of a moving object are nonrealistic, but if the changes in shape have features of the motion being depicted, they may be interpretable by the observer without a convention's needing to be explained.

Kennedy and Gabias (1985) tested blind and sighted subjects with five pictures of wheels with spokes, asking, for example, which of the pictures best suggested wobbly motion. The blind and the sighted both picked a picture with wavy (sinusoidal) spokes as best suggesting wobbly motion. Other motions included steady spinning, jerky motion, spinning too fast to make out, and a wheel with its brakes on. However, the pictures tested by Kennedy and Gabias were ones devised by the experimenters, not simple direct copies of ones made by the blind.

Kennedy (1993) reported several studies in which blind people were asked to make drawings of moving objects or were asked to interpret pictorial devices invented by the sighted, but none testing the recognizability of the drawings from the blind. Can devices invented by the blind to depict motion communicate effectively to the sighted?

To begin to answer this question, we asked a blind man who had shown interest in pictures to devise some pictures suggesting motions of various kinds. We asked him to draw a wheel with five spokes, showing six kinds of motion-related referents. We then tested the pictures with sighted subjects.

We asked our blind informant if he had ever drawn the referents we suggested prior to our test. He noted that he had not, and he expressed surprise at the selection of topics, but he gave the task some thought and then proceeded. We photocopied the pictures he drew (Figure 1). We presented the copies in two studies to sighted subjects, who were asked to select the relevant labels, in multiple-choice fashion. In Experiment 1, sighted subjects identified two groups of three displays accompanied by three labels. In Experiment 2, all six displays and labels were presented simultaneously.

\section{EXPERIMENT 1}

In this experiment, only three pictures and three options were presented at one time. The purpose of our first test was to determine whether the kind of motion intended could be identified in relatively undemanding circumstances.

\section{Method}

Subjects. The subjects were 24 undergraduate volunteers (mean age, 22 years; $S D, 2.8 ; 19$ female) from a class on perception, tested as a group. The project was one of several demonstrations for which members of the class could volunteer. The subjects were distributed around a lecture hall seating over 100 , with no subject having a fellow subject in an immediately neighboring seat. One volunteer who had some difficulties with small print had the instructions read to her, to help ensure comprehension of the instructions.
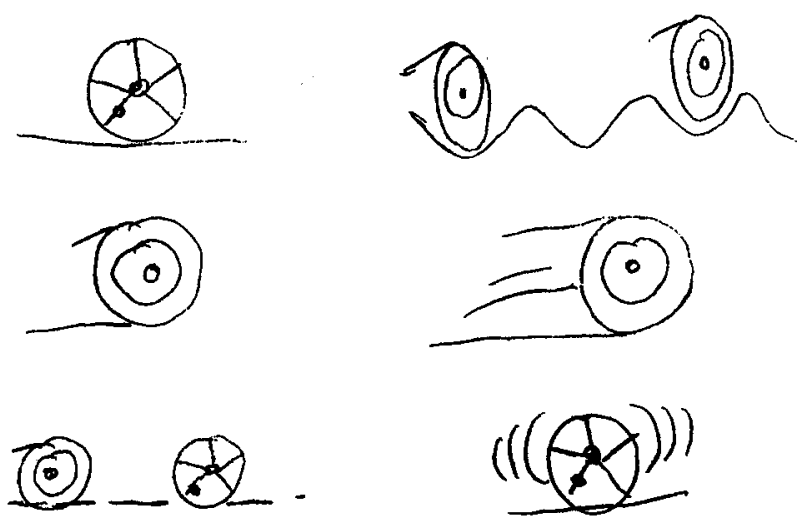

Figure 1. Six pictures drawn by E.A. In order, from the top left, reading down, they show a static wheel, a wheel spinning steadily, and $a$ wheel in jerky motion (Set $A$ ). From the top right, reading down, they show a wheel in wobbly motion, a wheel spinning too fast to make out, and a wheel with its brakes on (Set B). 
Stimuli. The subjects were shown two sets of drawings - Set A and Set B. One set had to be completed and sealed in an opaque envelope before the second, with its options, was shown. Each set had three pictures. Each set was presented on a $28 \times 21 \mathrm{~cm}$ sheet of paper. The top third of the sheet was one picture, the middle third a second, and the lower third the last picture of the set. The pictures ranged in size from 10 to $18 \mathrm{~cm}$ wide and from 3 to $6 \mathrm{~cm}$ tall.

In Set $\mathrm{A}$, one picture was our informant's response when asked to show a static wheel, one picture was intended to show a wheel in jerky motion, and one picture was to suggest a wheel in steady spinning motion. In Set B, one picture was to show a wheel with its brakes on, one a wheel in wobbly motion, and one a wheel going too fast to make out. For convenience, the pictures can be termed static steady, jerky, wobbly, too fast, and brakes on (brakes, in our tables).

The drawings were life-sized photocopies of drawings made by E.A., a congenitally totally blind man. E.A. has shown considerable interest in making pictures, although he received no formal instruction in depiction in any school. He regards himself as selftaught. He draws realistic pictures well, in a fashion quite recognizable by sighted people. Sighted people have remarked that they are surprised by the skill E.A. shows, and his pictures are highly commendable for anyone, sighted or blind. For example, we have seen pictures of a clown, a bowl of flowers, and houses by E.A. that are effortlessly recognizable. None of the pictures by E.A. that we have seen include devices for showing a wheel in motion, other than the wheel's being part of an object, such as a cycle, and E.A. reports that the task and his solutions were novel to him. When E.A. made the drawings, he was 44 years old. Our requests to E.A. were presented to him in his native language (Turkish) by J. Eroncel, who also translated his comments into English.

J. Eroncel has been familiar with E.A. and his pictures for several years. She informs us that, to her knowledge, E.A. has never drawn wheels in motion, using the devices evident in the pictures E.A. devised. She reports that these devices have not appeared in any pictures E.A. drew before, in her experience, and that, to her knowledge, E.A. has not been told about motion-indicating devices. She informs us that prior to our request to E.A., she had seen pictures of vehicles drawn by E.A., and none used the devices in Figure 1. After drawing the devices, E.A. described his source of ideas as direct contact with moving wheels, and he mentioned the wheels of children's bicycles or tricycles as relevant experiences. He noted that when the spoked wheels of these bicycles are turning, it is difficult to make out the individual spokes or small objects on the spokes. The drawings were made by E.A. with a pencil, on paper, using considerable pressure. The minor deformations in the paper are tangible. The lines E.A. made are clear in the photocopies shown to the subjects.

Procedure. Half the subjects tackled Set A first. The order of the three pictures in each set was varied. All the possible combinations of order of Set A (six orders) and Set B (six orders) were tested. All the possible orders of the three labels within a set (six orders each) were tested. No subject was given the labels to assign in an order that fitted the order in which the pictures were laid out. For each subject who was given the labels in the order $x, y, z$, there was a subject who received the labels in the order $z, y, x$. Also, each label was presented first an equal number of times.

\section{Results}

The subjects were offered three labels for each picture and were asked to select one. In principle, this would allow a subject to select the same label for each of the three pictures in that set. However, no subject offered that answer. In every case, the subjects selected a different label for each picture in each set. Accordingly, we have selected $1 / 6$ as the probability of assigning the labels correctly, assuming no replacement of labels once a
Table 1

Number of Subjects Assigning Three Labels Correctly to Pictures in Experiment 1

\begin{tabular}{lccc}
\hline Picture Set & First Set & Second Set & Total \\
\hline Set A & 10 & 12 & 22 \\
Set B & 7 & 9 & 16 \\
Total & 17 & 21 & 38 \\
\hline
\end{tabular}

label is assigned, rather than $1 / 27$, which is the probability of assigning the labels correctly if there is replacement.

The number of subjects assigning all three labels to the pictures in a set as E.A. intended $(p=1 / 6)$ is shown in Table 1 . In all the cells, the levels are above chance (binomial tests, $p<.01$ ).

A chi-square analysis reveals no significant differences between the four cells in Table $1\left[\chi^{2}(1, N=24)=\right.$ $.94, p>.05]$.

Tables $2 \mathrm{~A}$ and $2 \mathrm{~B}$ provide information on assignment errors. Set A induced few errors. Twice, the picture intended as static was labeled steady. What was intended as steady was called static once and jerky once. Once, the jerky picture was said to be static. In Set B, brakes on was assigned too fast five times, and correspondingly, for too fast, the chief error was assigning brakes on (six times). For wobbly there was no dominant error. It was assigned correctly 21 times and was twice called too fast and once brakes on.

Whereas 7 subjects made errors in the first set, only 3 subjects were in error on their second set, and no subject made errors on both sets.

\section{Discussion}

In this experiment, in which the subject's options were quite restricted, E.A.'s pictures conveyed their intended referents at a high rate. The results suggest that no subject was entirely insensitive to the meaning of the devices. The results in one of the four conditions were at ceiling (Set $\mathrm{A}$, as the second set attempted). In all three other conditions, the results were well above chance,

Table 2A

Frequency of Assignments of Labels to Pictures in Set A in Experiment 1

\begin{tabular}{cccc}
\hline & \multicolumn{3}{c}{ Assigned Label } \\
\cline { 2 - 4 } Intended Referent & Static & Steady & Jerky \\
\hline Static & 22 & 2 & 0 \\
Steady & 1 & 22 & 1 \\
Jerky & 1 & 0 & 23 \\
\hline
\end{tabular}

Table 2B

Frequency of Assignments of Labels to Pictures in Set B in Experiment 1

\begin{tabular}{cccc}
\hline & \multicolumn{3}{c}{ Assigned Label } \\
\cline { 2 - 4 } Intended Referent & Brakes & Wobbly & Too Fast \\
\hline Brakes & 17 & 2 & 5 \\
Wobbly & 1 & 21 & 2 \\
Too fast & 6 & 1 & 17 \\
\hline
\end{tabular}


never less than 7 perfect scores out of 12 , when the probable mean score was 2 by chance.

Two pictures were confused with each other relatively frequently: brakes on and too fast. To what should this be attributed? The confusion could occur because one of the pictures attracts several possible labels. Both are capable of specifying their referent to some extent, since the dominant response is correct for both. But one of the pictures may offer more ambiguity than the other. In our second study, we placed fewer restrictions on the subjects, which helped differences in recognizability to become apparent.

\section{EXPERIMENT 2}

Experiment 1 indicated that the displays were identifiable under favorable circumstances, with relatively few options. Experiment 2 was a more severe test of the recognizability of the pictures, providing all six options per picture, permitting a wider range of possible uses of each picture to be revealed.

\section{Method}

Subjects. A second group of 24 undergraduates (mean age, 22; $S D, 3.1 ; 20$ female), from a different class on perception than the first, volunteered for this experiment. They were tested individually. The project was one of several demonstrations for which class members could volunteer.

Materials. The pictures in Experiment 1 were used again in Experiment 2. All six pictures were presented in one envelope, and the six response options were given on the page with the instructions and response options. The pictures were on two pages, three per page, with the orders varied.

Procedure. Each subject was provided with a sheet of instructions containing the response options. Each option was labeled with a letter, $A$ to $F$. On the response sheet, the pictures were described as being on page number 1 or 2 , and as the top picture, the middle picture, or the bottom picture. The letters $A$ to $F$ followed each description. The subject was asked to circle a letter to indicate what the picture in a certain location was showing.

\section{Results}

Table 3 shows the frequency with which each label was assigned to each picture. Static was assigned its intended label 23 times, and wobbly and too fast were each assigned their intended labels 17 times. Steady was assigned that label 12 times. Jerky motion was given that label 10 times - but was identified as brakes on 12 times. The only picture rarely correctly assigned was the one intended as brakes on, which was labeled steady 9 times, wobbly 5 times, and jerky 4 times but only assigned brakes on 4 times. It was also called too fast twice but, interestingly, was never called static.

The subjects were not asked to assign the labels uniquely, one to each picture with no reassignment. However, 19 did so. Consequently, analysis can be based conveniently on these (see Table 4). Note that, for these subjects, it is impossible to obtain a score of five, since at least two labels must be misassigned.

The distribution of correct scores per subject is significantly different from chance $\left[\chi^{2}(5, N=19)=462.35\right.$,
Table 3

Frequency of Assignments of Labels to Pictures in Experiment 2

\begin{tabular}{cccccccc}
\hline & \multicolumn{6}{c}{ Assigned Label } \\
\cline { 2 - 7 } Intended Picture & Static & Steady & Jerky & Wobbly & Brakes & Too Fast \\
\hline Static & 23 & 0 & 0 & 0 & 1 & 0 \\
Steady & 2 & 12 & 4 & 0 & 3 & 3 \\
Jerky & 1 & 1 & 10 & 0 & 12 & 0 \\
Wobbly & 0 & 1 & 5 & 17 & 0 & 1 \\
Brakes & 0 & 9 & 4 & 5 & 4 & 2 \\
Too fast & 0 & 1 & 0 & 0 & 6 & 17 \\
\hline
\end{tabular}

$p<.0001]$. By way of illustration, by chance alone, the number expected to obtain scores as high as 3 is 1.5 , but the obtained frequency is 17 , much higher.

\section{Discussion}

Experiment 2 reinforces the lesson from Experiment 1 that the pictures convey their intention at a rate considerably above chance. The wheels intended as static, wobbly, and too fast provide high scores (all 17 or above). Two others - steady and jerky-perform well, considerably above the score of four expected by chance with no reassignment.

Since the motion-indicating devices induced scores far from ceiling in Experiment 2, the devices are equivocal to some extent. This point is made strongly by the results for the device that provided almost half the errors in Experiment 1. Brakes on is given its intended label at the chance rate of four (with no reassignment). In the presence of the labels for static, steady, and jerky, in addition to the labels accompanying it in Experiment 1 , it is frequently considered to be showing steady ( 9 times), and it is given all the other labels except for static a few times.

The results of both experiments suggest that the devices contain some features relevant to their intended referents but are ambiguous to a degree. Some of the devices may fit with Rosenblum et al.'s (1993) characterization of some devices as streak lines behind moving objects, but others involve omissions or nonliteral changes in the shape of the referents. The length of streak lines may be used aptly to specify motions related to intervals of time - that is, kinematics. In addition to being apt for the intended referents, the set may also contain features that could readily be interpreted as suggesting other referents. Rosenblum et al. suggested that some ambiguity arises when devices share features with more than one event. This proposal may be particularly relevant to the

\section{Table 4}

Number of Subjects Labeling Pictures Correctly in Experiment 2

\begin{tabular}{cc}
\hline Number of Pictures Assigned Correctly & Number of Subjects \\
\hline Six & 3 \\
Four & 5 \\
Three & 9 \\
Two & 1 \\
One & 1 \\
Zero & 0 \\
\hline
\end{tabular}


brakes on picture, which Experiment 2 showed was often not identified as intended.

Our concern in working with E.A. was to obtain devices and to test them, and a detailed interview on his intentions was not involved. In addition, some of the features to which the sighted subjects responded may not have been what E.A. envisaged. What we need to ask is what the sighted subjects may have been using.

The picture intended as a static wheel contains a wheel with five spokes, unadorned by other devices, except for a detail (a dot) on one spoke. Since it generally does not depart from plainly showing the perimeter as a circle and the five spokes, it shares with an actual static wheel the fact that the spokes are evident and details, such as a dot, can be made out. Also, the perimeter is not altered in shape from the true shape of the wheel. It is literally correct. It is the picture identified as showing its referent most frequently, likely because it has little in the way of a deviation from the norm of a wheel's shape that would call for some explanation. Similarly, a static wheel is one that has not deviated from rest, which might be taken to be the origin for a set of motions.

Consider now the features the pictures could be said to share with kinetic referents. To show steady spinning, one picture contains no spokes, but a circle is centered on the hub, and one streak line is at the top of the wheel. It also has a line at ground level on one side of the wheel. Deleting the spokes can suggest that they are not evident, as they are when the wheel is static. The unbroken circle in place of the spokes can suggest continuous motion and its path. The ground line can show the terrain covered in a unit of time.

Jerky motion is shown by two pictures, one apt for steady spin and one apt for a static wheel. The ground line is dashed, suggesting interruptions.

A wobbly motion is suggested by a drawing with two wheels underlined by a wavy line stretching between the two, which can suggest an undulating path of motion. Intriguingly, the wheel shape is made oval, its major axis vertical, echoing the up-and-down undulations. Both the wheels are turning, since both use the devices that were used to suggest steady spinning.

A wheel spinning too fast to make out is indicated by increasing the number of streak lines to three, by lengthening them, and by adding a long line at the base of the wheel. Lines especially long could suggest that the wheel moves a longish distance in a unit of time.

A wheel with its brakes on is drawn as a wheel showing its spokes and a detail on one spoke, accompanied by three arcs to the left and three to the right, symmetrically enclosing the wheel. The arcs may convey the wheel shuddering as it halts under brake power. The perimeter of the wheel would move to different locations if it was shuddering. The arcs may show the locations occupied by different parts of the perimeter at different times. The ground line is symmetrical, rather than mostly to one side, as in the case of the fast wheel, which can suggest there is no motion predominantly in one direction.

Although the pictures offer features relevant to their referents, they can be ambiguous. Brakes on has arcs on its left and right sides. The arcs are relatively smooth and even and are symmetrical. Steady spinning could be said to be smooth and even, and since it would entail no displacement to one side, it remains symmetrical. But also, a wheel in wobbly motion might be displaced first to the left, overlapping one arc, then to the right, aligned with another, then back to the left, and so on. If it moved rapidly from one arc's locus to another, it would be jerky and could be depicted by a wheel with arcs around it, suggesting some of the loci occupied in a unit of time. These options could help explain the many times-20 in all--the wheel was selected as something other than brakes on.

The picture intended as jerky involves two wheels, one with concentric circles and a trailing line, and one showing five spokes. Taken from left to right as a wheel in motion followed by a static wheel, this has the potential to show a wheel with its brakes on. Indeed, it is selected for brakes on slightly more often (12 times) than it is selected for jerky (10 subjects).

The wheel with concentric circles and a short trailing line was selected as steady much more than any other option. Having a short trailing line might be taken as suggesting abrupt motion. Jerky motion was indeed favored by 4 subjects.

The drawing with concentric circles and several long trailing lines was selected by 17 subjects as too fast, but also as brakes on by 6 subjects. The trailing lines could mean how far the wheel traveled in a unit of time (long meaning far), but also it could mean that this was the distance traveled in coming to a halt. Alternatively, these lines could suggest the strength of friction or the loudness of noises made as the brakes slam on. What is in common to all these referents is high intensity. That the number of streak lines increased from one to several, and each was relatively long, can suggest an event with especial intensity in a particular space.

The drawing with two elliptical wheels on wavy ground was intended as wobbly, but 5 subjects picked it for jerky. The pictures intended as jerky and wobbly both have two wheels. Wobbling means being in more than one place, and jerky means having more than one motion. Both have more than one component, which allows confusion.

Motions have many distinctive features, and pictures do too. The viewer is expected to select some of the common features of the motions and the pictures. But if each of the pictures has, say, five features and the motions also have several (say, four) and what is in common and relevant is two each time, it would be easy to select unintended features. For a match to be as intended, the relevant features have to be salient as well as present (Giora, Fein, \& Schwartz, 1998). Furthermore, the context of the 
match is a set of alternative pictures and a set of alternative options. These influence the attempt to pick out the salient features.

In illustrations, there is often a complex scene-landscape, cars, houses, animals, and so forth. These can often help dispel ambiguity and allow the observer to know what selection of possible motions is relevant and how features might best be used. Similarly, in language, indicating someone was hot would mean one thing in an argument, another in a contest, another in an embarrassing incident, and still another in the throes of romance. The features we select from the set that are in common between heat and emotion shift as the context alters. Metaphoric representation relies on this ability to change the pairings of features between a topic and a comparison, depending on context (Gibbs \& Gerrig, 1989; Lakoff \& Johnson, 1980). The key feature for "men are wolves" in a discussion about revenge could be "pack animals." In a casual comment about romance, it could be "lone hunters." This change with context is known as the problem of relevance (Sperber \& Wilson, 1995). Chiappe (1998) points out that a full treatment of the ways in which relevance is attained is beyond the reach of current cognitive science.

Spokes do not become circles when wheels revolve. But both spinning wheels and circles are continuous and involve similar shapes. Wobbly wheels are not vertically elongated ellipses, but the elongation can be in the direction the wheel moves when wobbling and, thereby, have a shape that suggests the wheel's path. Ground can be continuous but can be shown as dashed to suggest discontinuities in a motion. The pictures show the objects as if certain transformations were produced by motions. Temporal features of motion are transferred to the spatial properties of wheels. What is remarkable is that this transfer was made innovatively and effectively by a blind person, on being given the task of depicting motions.

The devices used by E.A. and understood by the sighted subjects here are unlikely to be found in pictures prior to the 19th century (Kennedy, Green, \& Vervaeke, 1993). It may be that the need to show machines with wheels that moved while remaining in one location was more pressing in that century than before. The wheels would appear immobile if they were simply drawn in detail, showing all the spokes. The advent of photography, and the interesting results of time-lapse photography, may have interested artists in the goal of drawing objects without detail, with devices such as blur and with evidence of paths of motion. Thus, the invention of these kinds of motion-indicating devices in the 19th century by sighted artists may have been the discovery of a way of depicting, and not the inventing of an arbitrary code. Outline makes sense without its needing to be explained, and motion indicators can be found by the blind and the sighted to have apt referents without each device's needing to be explained.
E.A. devised the present pictures at a few minute's notice. We suggest that his devices involve selection from salient features-albeit, with some idiosyncrasies. The path of the motion, the motion's changes in time, the shape of the wheel and its spokes are all salient, and the selection of these features is therefore to be expected. What is individual is surely the particular recombination of these features that E.A. devised and the introduction of some relevant but nonessential factors. It is unlikely that another individual would introduce and break a ground line, as E.A. did, to suggest jerky motion and would curve the ground line to suggest wobbly motion. Others might have used the shapes of the streak lines to show these motions. Physically, the modification of the ground line to show wobbly motion is a minor variation in the placement of a motion-indicating device, although the nonliteral use of the ground line is a nice example of the metaphoric nature of the pictures.

Our argument is that, as in any drawing task, some aspects of the productions here are likely to be general, and some highly individual. The motion paths and the changes in motion direction are defining features of the motions E.A. was attempting to convey. The wheel's circumference and spokes likewise are defining features of the target object. These should be general. The use of a ground line is not essential to either the motion or the object and, hence, would be less general, although it is relevant. Likewise, E.A.'s decision to use the number and length of trailing lines to show variation in speed is understandable, but clearly there are options. Variations in the thickness of the trailing lines or in the number of circles within the wheel might have served the same purpose, once variation in a dimension on the page was used to stand for variation in the referent. Particular combinations of the defining features and relevant nonessentials are more idiosyncratic than the selection of pertinent features. By way of analogy, sentences are apt combinations of relevant words. The selection used at a particular time is hard to predict and idiosyncratic, but the topic and the claim to be made do offer general constraints. Also, the basic decision to allow the pictures (or, in our analogy, sentences) to be metaphoric and to stray from the goal of making literal copies might not be made by some subjects.

Of course, the referents of any metaphoric or literal pictures being used by the blind and the sighted would need to be somewhat familiar to both the sighted and the blind. Metaphorical allusions to brakes via a picture can only make sense if these objects are understood in a similar fashion by the person offering the metaphor and the recipient. Metaphor relies on common ground.

The present results provide interesting leads for further inquiry. The age at which metaphoric pictures can be devised by the sighted or the blind is unknown. Likewise, there are unanswered questions about the developmental level or the level of expertise with pictures that 
we might need to reach before we can decide to abandon making literal copies and to try to devise metaphors that are successful in communicating their intention. An important question for future research is whether we know that a metaphoric picture we have just devised is likely to be taken by others as apt. Perhaps we can recognize that our metaphoric picture relies on highly salient, distinctive aspects of the referent and distributes them suitably and systematically, despite being nonrealistic. Alternatively, many devices may be idiosyncratic, appearing unequivocal to their inventors but opaque to anyone who is uninitiated. Similarly, speakers may offer ambiguous sentences without realizing how ambiguous they are. When we initiated this research, we were quite unsure how E.A. might respond and had no technique ready to assess his ideas about his pictures. It might prove valuable to query those making drawings about the meanings and ambiguities they envisage. Also, there are many kinds of contexts in which pictures can appear, and it is likely, therefore, that there are many possible effects on the interpretability of particular devices that need to be explained. The ambiguity of the brakes on picture in Experiment 1 became more evident as the range of options increased in Experiment 2. Furthermore, it is important to test more blind individuals to determine the extent to which E.A.'s abilities are representative. We expect that blind people who are interested in spatial tasks, like E.A., and who take to novel problems with a will, as E.A. does, are likely to realize that they could try to invent metaphoric solutions to our target referents, but others will simply deem their skills too limited, their knowledge of pictorial tactics too limited, or the referents impossible to depict. If they do try to invent metaphoric devices, we suggest that they will employ defining features of the object and the target motion but that the combinations they will devise will be quite varied. Further variation will be introduced by some individuals selecting relevant parts of a context for the motion, such as the ground. The range of referents for metaphoric devices remains to be established. Certain kinds of motion-for example, ones in the saggital plane or reversible motionsmay present distinctive problems. The history of the uses of metaphoric devices in various parts of the world is far from understood. Some may be widespread. Lakoff and Johnson (1980) suggest some spatial images are important for much of cognition. The present studies provide results that motivate studies on such topics.

In sum, devices that are apt but nonrealistic ways of showing their referents can be invented by a blind person and can be intelligible to the sighted. The devices use shapes of motion paths but locate them metaphorically, sometimes distorting true shapes, and vary the number, length, and continuity of lines in an effort to convey such aspects as faster and slower motion or continuous and discontinuous movements.

\section{REFERENCES}

ARnheIm, R. A. (1974). Art and visual perception. Berkeley: University of California Press.

CHIAPPE, D. (1998). Similarity, relevance and the comparison process. Metaphor \& Symbol, 13, 17-30.

D' Angiulli, A., Kennedy, J. M., \& Heller, M. A. (1998). Blind children recognizing tactile pictures respond like sighted children given guidance in exploration. Scandinavian Journal of Psychology, 39, $187-190$.

ERIKsson, Y. (1998). Tactile pictures: Pictorial representations for the blind, 1784-1940. Gothenburg: Gothenburg University Press.

Friedman, S. L., \& Stevenson, M. B. (1980). Perception of movement in pictures. In M. A. Hagen (Ed.), The perception of pictures (Vol. 1 , pp. 225-255). New York: Academic Press.

GiBBS, R. W., \& GerRIG, R. J. (1989). How context makes metaphor comprehension seem special. Metaphor \& Symbolic Activity, 3, 145158.

Giora, R., Fein, O., \& Schwartz, T. (1998). Irony: Graded salience and indirect negation. Metaphor \& Symbol, 13, 83-102.

HeLler, M. A. (1989). Picture and pattern perception in the sighted and the blind. Perception, 18, 379-389.

Heller, M. A., \& KenNedy, J. M. (1990). Perspective taking, pictures, and the blind. Perception \& Psychophysics, 48, 459-466.

Holmes, E., Hughes, B., \& Jansson, G. (1998). Haptic perception of tactual gradients. Perception, 27, 993-1008.

Kennedy, J. M. (1993). Drawing and the blind. New Haven, CT: Yale University Press.

KENNEDY, J. M. (1997, January). How the blind draw. Scientific American, 276, 60-65.

KenNedy, J. M., \& Gabias, P. (1985). Metaphoric devices in drawings of motion mean the same to the blind and the sighted. Perception, 14 . 189-195.

Kennedy, J. M., Green, C. D., \& Vervaeke, J. (1993). Metaphoric thought and devices in pictures. Metatphor \& Symbolic Activity, 8 , 243-255

Kim, H., \& Francis, G. (1998). A computational and perceptual account of motion lines. Perception, 27, 785-797.

Lakoff, G., \& Johnson, M. (1980). Metaphors we live by. Chicago: University of Chicago Press.

MiLLAR, S. (1991). A reversed lag in the recognition and production of tactual drawings: Theoretical implications for haptic coding. In M. A. Heller \& W. Schiff (Eds.), The psychology of touch (pp. 301-325). Hillsdale, NJ: Erlbaum.

MILLAR, S. (1994). Understanding and representing space: Theory and evidence from studies with the blind and sighted. Oxford: Oxford University Press.

MILLAR, S. (1997). Reading by touch. London: Routledge

Rosenblum, L., Saldana, H., \& Carello, C. (1993). Dynamical constraints on pictorial action lines. Journal of Experimental Psuchology: Human Perception \& Performance, 19, 381-396.

Runeson, S., \& FryKholm, G. (1981). Visual perception of lifted weight. Journal of Experimental Psychology: Human Perception \& Performance, 7, 733-740.

SPERBER, D., \& Wilson, D. (1995). Relevance: Communication and cognition. Cambridge: Cambridge University Press.

(Manuscript received February 1, 1999; revision accepted for publication December 20, 1999.) 